\title{
Normal Pressure Hydrocephalus with Onset Following a Traumatic Experience
}

\author{
Aishatu Yusha'u Armiya'u \\ Department of Psychiatry, Jos University Teaching Hospital, Jos. Plateau state, Nigeria \\ *Corresponding author: Aishatu Yusha'u Armiya'u, Department of Psychiatry, Jos University Teaching Hospital, Jos. Plateau state, Nigeria, Tel: +2348035899338; E- \\ mail: aarmiyau@gmail.com; azuminiarmiyau@yahoo.com
}

Rec date: Aug 23, 2014, Acc date: Oct 19, 2014, Pub date: Oct 21, 2014

Copyright: (c) 2014 Armiya'u AU. This is an open-access article distributed under the terms of the Creative Commons Attribution License, which permits unrestricted use, distribution, and reproduction in any medium, provided the original author and source are credited

\begin{abstract}
Background: Normal Pressure Hydrocephalus (NPH) is a neurological disorder caused by excessive accumulation of cerebrospinal fluid (CSF) with resultant pressure to the brain. It is a rare syndrome usually found in the sixth and seventh decades of life. The condition is often misdiagnosed because of its non specific symptoms and chronic nature, but recently NPH is receiving a great deal of media and medical attention, leading to a more efficient and timely diagnosis and treatment.
\end{abstract}

Methods: This is a case report of a 40 year old widow, who presented with eight weeks history of recurrent vomiting, fearfulness and withdrawal to self following a traumatic experience. Prior to above presentation patient was normal and stable with no past history of physical or mental illness.

Results: Clinical features met the criteria for diagnosis of Post Traumatic Stress Disorder following a traumatic experience. Clinical examination and investigations including (skull xray) conducted were all within normal limits. Two weeks into admission patient developed seizure, facial nerve palsy, and progressive weakness of lower limbs which warranted further investigation. The patient had Computer Tomography scan which revealed NPH, she had a ventro-peritoneal shunt at the neurosurgical unit of the hospital.

Conclusion: Despite the advances in the diagnosis and treatment of hydrocephalus, there is still much to be learned about hydrocephalus and the subtle forms it can take. Even though it is more common in infants and elderly, it can occur at any time in life and as a result of a variety of causes.

\section{Introduction}

Hydrocephalus is primarily characterized by excessive accumulation of fluid in the brain [1]. Normal Pressure Hydrocephalus (NPH) is a type of hydrocephalus which is caused by decreased absorption of CSF. This leads to an increase in the brain pressure due to excessive accumulation of CSF in the ventricles of the brain. Majority of the patients with NPH are elderly 60 years and above. In developing countries such as Nigeria managing patients with $\mathrm{NPH}$ is hindered significantly because of economic constraints [2]. The condition has several etiological causes which include following a stroke, bleeding around the brain, head injury, brain tumors or meningitis. Irrespective of the etiological cause of NPH, CSF shunts are used to treat the condition though the procedure is expensive and not always available [3]. The management of hydrocephalus and associated complications of its treatment warrants considerable surgical judgement and a life-long approach to patient's follow-up [3].

\section{Case Report}

A 40 year old widow and house wife Mrs. L.C.B was referred from the General Outpatient Department of Jos University Teaching Hospital to the psychiatric unit of the same hospital on the 22nd of May 2014, she presented with eight weeks history of recurrent headache, recurrent vomiting, fearfulness and withdrawal to self. She was in good state of health until eight weeks prior to presentation when she witnessed the crisis in Wukari Yobe state, Nigeria. She was reported to have experienced repeated gun shots following which she complaint of headache. Headache was generalized; throbbing, and non-radiating, headache was relieved mildly following ingestion of analgesic, but present almost every day. There was associated blurring of vision and dizziness which is worse when she attempts to walk. At about the same time she complaint of intermittent fever, worse in the morning with associated rigors but no chills. Fever was relieved by ingestion of antipyretic. No associated neck stiffness or convulsion. Over the previous two weeks she was said to feel weak and could walk only with support.

During admission, patient had three to four episodes of vomiting per day, which were initially projectile, not associated with food, but associated with nausea, crampy sensation in the abdomen and loss of appetite. No abdominal pain, swelling, or change in bowel habits. No pain difficulty on micturition, or change in frequency of micturation. No cough, chest pain or breathlessness.

She was withdrawn to self, not interacting with her family as she used too. Reported to think a lot about her horrific experience at Wukari and often experience ringing sensation in her head. She had intrusive recollection of the event, nightmares, and flashbacks. When asked about the event she was unable to recall some aspects of the event, loss interest in pleasurable activities, though she had no significant change in her mood. She experienced startle reactions and finds it difficult to concentrate. This was the first episode of mental illness in the patient, and she had no previous history of medical problems. No previous history of trauma, accident or surgical interventions. No family history of mental illness. No history of 
alcohol or other psychoactive substance use. She is reported to be an extroverted, jovial person with a predominant cheerful mood. She had a lot of friends, not easily angered, hardworking and religious.

A middle aged woman, tall, slim built, fair in complexion, in fair nutritional state, kempt, and wore traditional attire. She was brought into the consulting room supported by relatives, fairly cooperative and maintained good eye contact. Her speech was responsive, coherent, answered to questions directed at her, reduced tone, and normal volume. Mood was normal (as reported by patient), affect was restricted. Her thought was free flowing with no formal thought disorder; thought content was preoccupied by her illness. No perceptual abnormalities. She was conscious and alert, oriented in place and person but not in time. Attention and concentration were impaired. On physical examination she was warm to touch (temp 36.5 degree centigrade), not pale, anicteric, acyanosed, mildly dehydrated, no significant peripheral lymphadenopathy, and no pedal oedema. All other systems were normal on examination.

A diagnosis of Post-Traumatic Stress Disorder (PTSD) was made to rule out septicemia. Treatment was commenced: pharmacotherapy antidepressant tablet paroxetine $20 \mathrm{mg}$ daily; antibiotics and antimalarial; psychotherapy using Cognitive Behavioural Therapy (CBT). Results of investigations were all within normal limits which include (Full Blood Count and differentials, Fasting Blood Sugar, Urea/ Electrolyte/Creatinine, Liver Function Test, Blood Culture, Urine Culture, Urinalysis and Skull $\mathrm{x}$-ray.

Two weeks into admission at the psychiatric unit, she was reported to have convulsed. Convulsion was tonic clonic in nature with associated upward rolling of the eye balls. At about the same time patient complaint of progressive weakness of the lower limbs bilaterally which prevented her from mobilizing, as well as urinary incontinence both during the day and night. A times her speech was irrational, had visual hallucination with altered level of consciousness. Central nervous system examination was conducted and patient was found to be conscious and alert but lethargic, she was oriented only in person not in place and time, right and left upper limbs were grossly normal but lower limbs had reduced muscle tone and reflexes with power of $2 / 5$ bilaterally. At that point a diagnosis of Generalized Tonic-Clonic seizure (GTCS) was made to rule out intracranial space occupying lesion and an electroencephalograph (EEG) investigation was requested, which confirmed the diagnosis of GTCS. Anticonvulsant medication tablet carbamazepine $200 \mathrm{mg}$ was commenced twice daily with low dose antipsychotic tablet trifluoperazine $5 \mathrm{mg}$ at night for hallucination. Due to financial constraint patient could not afford a CT scan or MRI.

Monies where raised for CT scan and the scan result revealed communicating normal pressure hydrocephalous (NPH). She was referred to the team of neurologist and neurosurgeon. She had ventriculo-peritoneal (v-p) shunt surgery at the Jos University Teaching Hospital. Following the surgery she did well and all symptoms subsided and she was able to mobilize with minimal support before her discharge. She was lost to follow-up.

\section{Discussion}

The prevalence and incidence of hydrocephalus in developed nations is estimated at $0.9-1.2 / 1000$ and $0.2-0.6 / 1000$ respectively [4]. An estimate for Africa is not reliable, but its incidence is likely higher and mostly found among untreated or poorly treated neonatal meningitis and nutritional deficiencies [3]. An extrapolated incidence of 168 using a population estimate of $125,750,356$ was found for Nigeria [5]. For NPH recent estimates from population based studies reported a prevalence rate of $0.5 \%$, an incidence of about 5.5/100,000 patients in those over 60 years old per year [6,7]. On presentation patients with NPH present with gradual progressive disorder noted by the classic triad consisting of abnormal gait, urinary incontinence and dementia, the triad was first described in 1965 by Hakim and Adams [8]. In NPH due to the expansion of the ventricular system especially the lateral ventricles gait abnormality is the most prominent symptom and is characterized as bradykinesia, broad based, magnetic and shuffling. Urinary incontinence presents as urinary frequency, urgency, or frank incontinence. While incontinence may result from gait disturbance and dementia, Sakakibara et al. in their study found that $95 \%$ of their patients had urodynamic parameters consistent with detrusor over activity [9]. The average time of diagnosis which is the time between onset of symptoms and a diagnosis of hydrocephalus appears to be significant, and is often years [9].

The diagnosis of Mrs. L.C.B was made by radiological investigation precisely Computer Tomography scan (CT scan) within four weeks (Figure 1). The age of patient, time of presentation and clinical picture of patient were not in tandem with NPH. Prior to presentation patient was stable and doing well, her symptoms were triggered by the traumatic experience she had at Wukari; those symptoms met the criteria for PTSD. The gait abnormality was due to weakness of the lower limbs, which results from progressive expansion of the ventricular system; urinary incontinence was evident after two weeks, which was likely due to a decrease in the inhibition of bladder contraction and instability of the detrusor but the patient had no dementia [10]. The convulsion Mrs. L.C.B had while on admission could likely be due to brain compression as a result of accumulating fluid which led to the neurological symptom of convulsion or epileptic seizure [11]. This signs occur earlier in adults because the adult skull no longer expand to accumulate the increasing volume of fluid within [11]. All clinical examinations and investigations done earlier were within normal limits. Because of the patients behaviours and diagnosis of PTSD neuropsychological assessments were done, so also intensive interview for the patient and family members. She had series of test to assess her cognitive function which include memory, attention and executive functioning. Hydrocephalus can result in brain injury, as such thought processes and behaviour of the patient may be affected adversely. Therefore in Mrs. L.C.B's case the ultimate goal of treatment was to understand how changes in her brain structure and function affected her behaviour. These changes were found to be as a result of $\mathrm{NPH}$ which caused the neurological abnormalities seen in the patient. Following the shunt she recovered and was able to mobilize and comprehend well. A study in 2004 found that $86 \%$ of patients had beneficial outcomes after shunt insertion, which is observed in either improved incontinence (70\%), gait disturbance (81\%) or both [12]. Although she was lost to follow-up the managing team was happy because she was treated of her ailment successfully by three specialists (Neuropsychiatrist, neurologist and neurosurgeon).

The unique and confusing nature of hydrocephalus in young and middle aged adults, makes it exceedingly different from hydrocephalus diagnosed in infancy, early childhood, or that found in older adults (typically 60 years and older) [13]. Recently it has began receiving attention formally from the medical community, in this age group hydrocephalus presents a host of challenges and opportunities for both patients and medical professionals alike. These challenges go beyond specialized medical care, but also include psychological, emotional and 
Citation: Armiya'u AY (2014) Normal Pressure Hydrocephalus with Onset Following a Traumatic Experience. J Neurol Disord 2: 184. doi: $10.4172 / 2329-6895.1000184$

Page 3 of 3

occupational issues all of which are important for the holistic management of the patient [13].

\section{Conclusion}

Normal pressure hydrocephalus can be relieved successfully with a shunt implanted surgically for the drainage of the excessive cerebrospinal fluid. Care need to be taken to prevent malfunctions of such shunts, because this will likely result in infection, which occur in several cases. Occurrence of this warrants prompt action which should be taken immediately. For a successful hydrocephalus treatment, it is important to establish relationship with the health care team. Communication and trust are good components for a successful relationship and a holistic treatment.

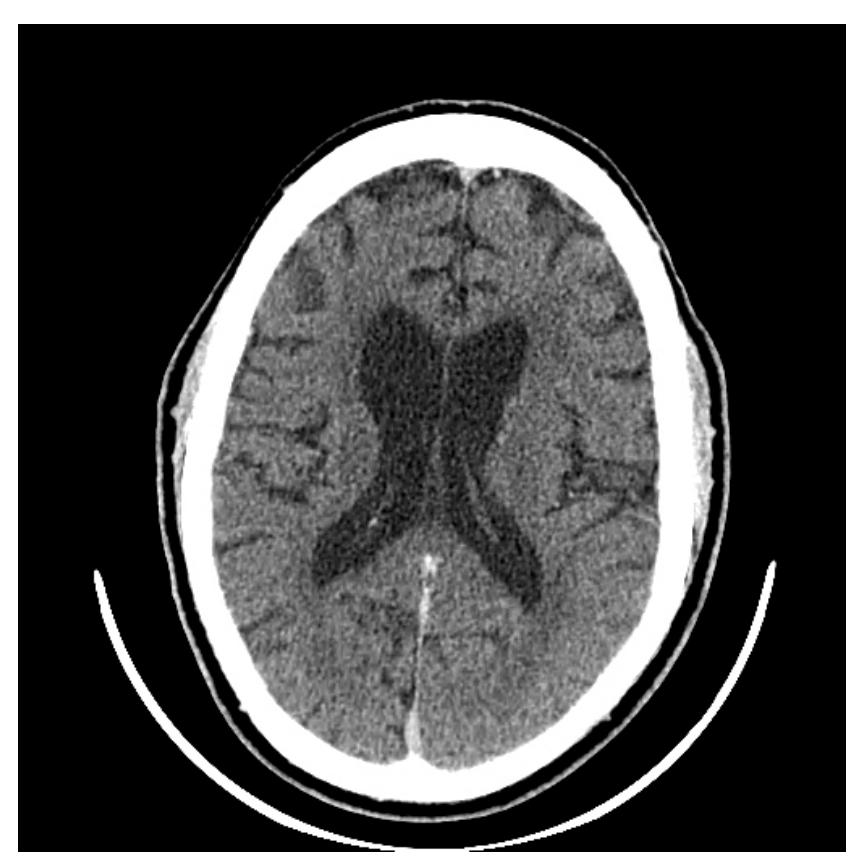

Figure 1: CT scan of Mrs. L.C.B

\section{References}

1. National Institute of Neurological Disorders and Stroke (2013) Hydrocephalus facts sheet. NINDS Publication. 08: 385.

2. Warf BC (2005) Hydrocephalus in Uganda: the predominance of infectious origins and primary management with endoscopic third ventriculostomy. Journal of Neurosurgery (Pediatrics 1) 102: 1-15.

3. Bauman N, Poenaru D (2008) Hydrocephalus in Africa: A surgical perspective.

4. Garton H, Piatt J (2004) Hydrocephalus. Journal of Pediatric Clinics N.A. 51: 305-325.

5. US Census Bureau (2004) International Data Bank.

6. Brean A, Eide PK (2008) Prevalence of probable idiopathic normal pressure hydrocephalus in a Norwegian population. Acta Neurologica Scandinavica 118: 48-53.

7. Tanaka N, Yamaguchi S, Ishikawa H, Ishii H, Meguro K (2009) Prevalence of possible idiopathic normal pressure hydrocephalus in Japan. Neuroepidemiology 32: 171-175.

8. Adams RD, Fisher CM, Hakim S, Ojemann RG, Sweet WH (1965) Symptomatic Occult Hydrocephalus with Normal Cerebrospinal-Fluid Pressure. New England Journal of Medicine 273: 117-126.

9. Sakakibara R, Uchiyama T, Kanda T, Uchida Y, Kishi M, et al. (2008) Urinary dysfunction in idiopathic normal pressure hydrocephalus. Brain Nerve 60: 233-239.

10. Younger DS (2005) Adult normal pressure hydrocephalus. In Motor disorders (2nd edn), Lippincott Williams \& Wilkins, Philadelphia, USA.

11. Poca MA, Mataro M, Matarin M, Arikan F, Junque C, et al. (2004) Is the placement of shunts in patients with idiopathic normal pressure hydrocephalus worth the risk? Results of a study based on continuous monitoring of intracranial pressure. Journal of Neurosurgery 100: 855-866.

12. Aschoff A, Kremer P, Hashemi B, Kunze S (1999) The scientific history of hydrocephalus and its treatment. Neurosurgical Review 22: 67-93.

13. Rekate HL, Williams MA (2003) Hydrocephalus Diagnosed in Young and Middle-Aged Adults. A Book for Adults and their Families (2nd edn), San Francisco, California, USA. 